\title{
A Comparative Cross-sectional Study on Anthropometry and Body Composition of Selected Female Type 2 Diabetics and Non-diabetics
}

\author{
Bhavani S and Jemima Beryl Mohankumar* \\ Department of Nutrition and Dietetics, PSG College of Arts and Science, Coim- \\ batore, TN, India \\ *Corresponding Author: Jemima Beryl Mohankumar, Department of Nutrition and \\ Dietetics, PSG College of Arts and Science, Coimbatore, TN, India.
}

Received: November 02, 2020

Published: November 18, 2020

(C) All rights are reserved by Bhavani $\mathrm{S}$ and

Jemima Beryl Mohankumar.

\begin{abstract}
A study was designed to compare the body composition of female diabetics and non-diabetics in the age group of 35 - 45 years. A validated questionnaire was used to collect background information. The tools used to measure anthropometric measurements were properly calibrated and used according to the NHANES protocol. The Waist-to-Height Ratio (WHtR) and Body Adiposity Index (BAI) are focused in this article. Other anthropometric measurements are also presented. We found BMI to be significantly different ( $\mathrm{p} \leq$ 0.05 ) between the diabetics and non-diabetics. The diabetic women had greater subcutaneous fat in the trunk and legs. The skeletal muscle percentage was also lower. We recommend that body composition may be studied using the hand-held BIA device to make a better assessment of the metabolic condition to suggest suitable management.
\end{abstract}

Keywords: T2DM; WHtR; BAI; Body Composition; Sub-cutaneous Fat

\section{Introduction}

The burden of diabetes has steadily increased over the past quarter century in India and across the globe, with India contributing a major part of the global burden. Diabetes was identified as one of four priority non-communicable diseases (NCDs) targeted for action by the United Nations due to its growing disease burden. Physical activity helps to improve the body's response to insulin which can lower blood glucose levels, lower blood pressure and cholesterol levels, reducing the risk of heart disease, control weight, reduce the risk of developing diabetes complications [1].

Simple anthropometric measurements have been used as surrogate measurements of obesity and have more practical value in both clinical practice and for large-scale epidemiological studies [2]. BMI is a simple method which is used to calculate the prevalence of overweight and obesity in the population. Waist circumference (WC) is the best measure of both intra-abdominal fat mass and total fat [2]. But BMI can be misleading, such as in individuals with a high proportion of lean muscle mass [3]. WC, a more accu- rate measure of the distribution of body fat, has been shown to be more strongly associated with morbidity and mortality [3]. Recently, the waist-to-stature ratio (WSR) has been proposed as a better screening tool than WC and BMI for adult metabolic risk factors [4].

While there are several research endeavors and journal articles on the conventional anthropometric measurements the world over and even among South Asians, we did not find any on waist to height ratio and body adiposity index among Indians. Therefore, in this article we present our findings which we believe is the first of its kind.

\section{Materials and Methods}

PSG - Periya Veedu or Peelamedu Samanaidu Govindasamy. The study was conducted at PSG Institute of Medical Sciences and Research, Peelamedu, Coimbatore, India which has an Institutional Human Ethics Committee. This committee is registered with the Institutional Review Board Organizations IORG of Office for Human Research Protections OHRP, United States Department of Health 
\& Human Services. Ethical clearance was obtained to conduct the present study. The study was conducted in the out-patient facility of the Endocrinology Department of the same hospital.

Sample size was calculated using the online facility www.http// surveysystem.com by using data on current adult population of Coimbatore, number of type 2 diabetics, prevalence rate and $95 \%$ confidence level. The study participants were selected on the bases of inclusion viz. all the participants were the children of type 2 diabetics. They were women diabetic ( $\leq 5$ years) and Non-Diabetics in the age group of 35 - 45 years. Those excluded were the ones' with Sarcoidosis, kidney diseases, Systemic disease, Early menopause, HRT, usage of medicines for corticosteroids, diuretics, blood pressure and persons with Hypothyroid, hyperthyroid, Cushing syndrome, primary hyperparathyroidism, renal failure, liver disease, inflammatory bowel disease, malabsorption syndrome, alcoholism, osteoporotic breakage history and scoliosis.

The study design

Our study was an observational study, as the study participants were in a non-controlled environment without actually interfering or manipulating with other aspects of life and therefore is non-experimental. The observations were current and at a specified point of time. We can further describe our study design as a case-control study as we tried to determine the degree of associations of the selected parameters between diabetic and non-diabetic. Typically, we identified appropriate representative controls for the cases that we were studying [5].

\section{Data collection}

Baseline data was collected using a validated questionnaire. Anthropometric measurements were recorded with the help of the physician assistant. A properly calibrated electronic-digital scale, Omron Digital Body Weight Scale HN-283, Singapore purchased locally was used to measure the weight of the participants. Height measurements were taken using a 'drop down' non-stretchable tape measure fixed at about two meters on the wall. Waist circumference was measured according to the protocol given by National Health and Nutrition Examination Survey NHANES [6] and recorded to the nearest $0.1 \mathrm{~cm}$. Waist Circumference (in $\mathrm{cm}$ ) is divided by Body Height (in cm) is WHtR. A boundary value of WHtR $=0.5$ indicates increased health risk for men and women [7].

Fat mass and percent fat mass were estimated using a hand-held BIA (bio-electrical impedance analysis) device (Omron HBF375,
Omron Healthcare, Europe). The amount and nature of body water affects the validity of most techniques especially BIA. For this reason, the specified guidelines were followed in preparation for assessment. Subjects were asked to fast for 3 hours, refrain from strenuous exercise for 12 hours, and empty their bladders $30 \mathrm{~min}$ prior to measurement. Subjects stood in an upright position and held the BIA device with both hands at $90^{\circ}$ away from their body. Each measurement was performed in triplicate by the researcher. Fat-free mass was calculated by subtracting each individual's fat mass from her respective body weight. Percent fat free mass was calculated by subtracting percent fat mass from $100 \%$ for each subject $[6,8]$. The BAI estimates percentage adiposity directly as given below.The BAI measure was validated in the "Triglyceride and Cardiovascular Risk in African-Americans (TARA)" study of African Americans. Correlation between DXA-derived \%adiposity and the BAI was $R=0.85$ for TARA with a concordance of $\mathrm{C}_{-} \mathrm{b}=0.95$. BAI can be measured without weighing, which may render it useful in settings where measuring accurate body weight is problematic. In summary, we have defined a new parameter, the BAI, which can be calculated from hip circumference and height only. It can be used in the clinical setting even in remote locations with very limited access to reliable scales. The BAI estimates \%adiposity directly.

$\mathrm{BAI}=\frac{\text { Hip }}{\text { Height }^{1.5}}-18$

Data were entered and analyzed using the SPSS version 25. Quantitative data were expressed as mean, median, standard deviation and 95\% confidence interval (CI) was calculated. The ' $t$ ' value was used to compare the two groups of study participants. For each ' $t$ ' value the ' $p$ ' value is also given to indicate that the sample data is not by chance.

\section{Results}

The cutoff value for normal BMI for men and women was $23 \mathrm{~kg} /$ $\mathrm{m}^{2}$. The cutoff values for WC and WHR were lower in women than in men. The values were significantly lower compared with the corresponding values in white populations [9]. This clearly shows that universal standards are not suitable for different population groups to assess the status of health and to decide when interventions are required to correct the health problem. Therefore, in our study we used the WHtR and BAI in type 2 diabetics and non-diabetics.

Independent samples t test; Confidence interval 95\%

We found a significant difference in the BMI between the diabetic and non-diabetics $(\mathrm{p} \leq 0.05)$. Body fat percentage was higher

Citation: Bhavani S and Jemima Beryl Mohankumar. "A Comparative Cross-sectional Study on Anthropometry and Body Composition of Selected Female Type 2 Diabetics and Non-diabetics". Acta Scientific Women's Health 2.12 (2020): 16-20. 
among the diabetic participants but not significantly different $(\mathrm{p}$ $\geq 0.05$ ). The waist circumference of diabetics was higher, but not significantly. The data on body composition given in table 2 will reveal greater detains on the fat distribution. This is typical of Asian Indians due to their short stature. All other parameters like weight.

Body fat distribution changes according to menopausal status, with central obesity more pronounced in postmenopausal women [10]. Studies have reported heterogeneity of human body fat distribution across racial groups [11,12].

\begin{tabular}{|l|c|c|c|c|}
\hline Indices & Diabetic & Non-diabetic & t value & p value \\
\hline Height $(\mathrm{cm})$ & $155 \pm 6.36$ & $156.9 \pm 4.9$ & -1.029 & 0.310 \\
\hline Weight $(\mathrm{Kg})$ & $64.14 \pm 6.75$ & $61.27 \pm 6.38$ & 1.427 & 0.161 \\
\hline BMI & $26.74 \pm 3.05$ & $24.9 \pm 2.4$ & 2.161 & 0.037 \\
\hline Waist $(\mathrm{cm})$ & $100.86 \pm 9.86$ & $95.85 \pm 10.29$ & 1.608 & 0.116 \\
\hline Hip(cm) & $104.73 \pm 10.8$ & $103.15 \pm 9.38$ & 0.56 & 0.616 \\
\hline WHR & $0.97 \pm 0.082$ & $0.93 \pm 0.086$ & 1.391 & 0.172 \\
\hline WHtR & $0.65 \pm 0.75$ & $0.611 \pm 0.72$ & 1.761 & 0.086 \\
\hline BAI\% & $36.41 \pm 6.47$ & $34.68 \pm 6.36$ & 0.873 & 0.388 \\
\hline
\end{tabular}

Table 1: Means of anthropometric measurements.

\begin{tabular}{|l|c|c|c|c|c|c|}
\hline \multirow{2}{*}{ Indices } & \multicolumn{2}{|c|}{ Diabetics (n = 22) } & \multicolumn{2}{c|}{$\begin{array}{c}\text { Non-Diabetics } \\
\text { (n= 20) }\end{array}$} & \multirow{2}{*}{ t Value } & \multirow{2}{*}{ p Value } \\
\cline { 2 - 6 } & Mean & SD & Mean & SD & & \\
\hline Body fat (\%) & 33.31 & 5.00 & 31.42 & 4.44 & 1.303 & 0.200 \\
\hline Visceral fat (\%) & 14.95 & 4.65 & 12.60 & 3.59 & 1.845 & 0.073 \\
\hline BMR (kcal) & 1349.59 & 93.72 & 1507.00 & 102.90 & -5.165 & 0.000 \\
\hline Body age (years) & 58.96 & 17.09 & 47.65 & 11.01 & 2.573 & 0.014 \\
\hline Subcutaneous fat (\%) & 32.51 & 5.00 & 31.30 & 4.73 & 0.808 & 0.424 \\
\hline Whole body & 25.37 & 9.14 & 19.46 & 6.70 & 2.406 & 0.021 \\
\hline Trunk & 39.80 & 12.38 & 33.47 & 11.56 & 1.714 & 0.095 \\
\hline Arm & 42.24 & 10.41 & 34.82 & 10.47 & 2.302 & 0.027 \\
\hline Leg & 22.60 & 4.04 & 25.84 & 4.88 & -2.331 & 0.025 \\
\hline Skeletal muscle (\%) & 17.34 & 4.92 & 20.01 & 4.60 & -1.816 & 0.077 \\
\hline Whole body & 23.17 & 7.61 & 24.46 & 3.83 & -0.705 & 0.486 \\
\hline Trunk & 36.15 & 5.96 & 36.94 & 5.06 & -0.465 & 0.645 \\
\hline Arm & & &
\end{tabular}

Table 2: Comparison of mean body composition components.

Studies have also reported that Asian women carry greater abdominal and visceral fat when compared with Caucasian women with similar overall adiposity $[13,14]$. Thus, greater central relative to leg adiposity may explain why Asian women have a greater risk of diabetes compared with other race and ethnic groups. Results for Asian women suggested lower optimal cut points for anthropometric measures than other race/ethnicity groups [15].

Independent samples t test; Confidence interval 95\%

Data presented in table gives new insight into the body composition of diabetics. Though all selected study participants were in the age range of 35 to 45 years, the body composition study shows that diabetics were $58.96 \pm 17.09$ years. The difference was significant ( $p \leq 0.05$ ). The non-diabetics were only $47.65 \pm 11.01$ years. Other body composition indices that were significantly different include subcutaneous fat in the trunk and legs. With respect to skeletal muscles the whole body and trunk were different. Our observations indicate these changes in anthropometric indices and body composition may be the cause for the precipitation of the condition. Both the diabetic and non-diabetic group had one parent who was diabetic. However, we did not confirm if both parents diabetic. 
Obesity associated metabolic diseases have reached epidemic levels in many South Asian countries. Conventional anthropometric indices have poor sensitivity and specificity for detecting people with increased metabolic risks. Jayawardana, et al., [16], compared WHtR (Waist to Height Ratio) as a marker of diabetes and cardiometabolic risks with existing classical anthropometric indices such as; Body Mass Index (BMI), Waist Circumference (WC) and Waist to Hip Ratio (WHR) in a large sub-population of ethnic South Asians. A total of 5000 (Sri Lankans) subjects were recruited from a nationally representative community-based sample using multi-stage random cluster-sampling method. Anthropometric, biochemical and clinical parameters were measured. Receiver-operating characteristic (ROC) curves were performed and area under the curve (AUC) was calculated for each anthropometric index.

Data was analyzed for a sample size of 4485 Sri Lankans. The mean WHtR in all adults was $0.496( \pm 0.077)$, males $(0.477 \pm 0.065)$ had a significant lower WHtR than females $(0.508 \pm 0.081)(\mathrm{p}<$ $0.001)$. WHtR had the highest correlation with metabolic parameters. In all adults, males and females the AUC of WHtR was significantly higher than that of BMI, WC and WHR in diabetes mellitus, pre-diabetes, hypertension, metabolic syndrome and hypercholesterolemia. Mean age, fasting blood glucose, 2-h post prandial blood Glucose, total cholesterol, LDL cholesterol, triglycerides, systolic blood pressure and diastolic blood pressure were all significantly higher among all adults, males and females with WHtR $\geq 0.5$. They concluded that WHtR is a simple and effective anthropometric index to identify obesity associated metabolic risks among Sri Lankan adults [16].

Qualitative analysis and determination of visceral fat (VF) is more important [17] and bio-electrical impedance analysis (BIA) is a simple, quick, cost-effective and objective method available to assess the same with proven efficacy in Indian population and can be used on large scale even by family physicians and primary care providers [18].

\section{Conclusion}

From our study we would like to conclude that BMI is a sensitive indicator to assess the risk of diabetes. The body composition study gives more details of the alterations that may lead to the metabolic disorder. The data on body composition may be useful to draft a personalized management programme and to monitor the progression of the condition. Since our study was on women in the age group of 35 - 45 years, several of the changes in body composition may be attributed to the pre-menopausal stage. This requires further investigation with data on menopause.

\section{Note}

This article is a part of the Ph.D. thesis in Food and Nutrition submitted to the Bharathiar University, Coimbatore, Tamil Nadu, India. This research did not receive any funds from agencies that promote research.

\section{Bibliography}

1. Colberg SR. "Key points from the Updated Guidelines on Exercise and Diabetes". Frontiers in Endocrinology 8 (2017): 1-7.

2. Padaki S., et al. "Anthropometry and physical fitness in individuals with family history of type-2 diabetes mellitus: A comparative study". Indian Journal of Endocrinology and Metabolism 15 (2011): 327-330.

3. Dagan SS., et al. "Waist circumference vs body mass index in association with cardiorespiratory fitness in healthy men and women: a cross sectional analysis of 403 subjects". Nutrition Journal 12 (2013).

4. Browning LM., et al. "A systematic review of waist-to-height ratio as a screening tool for the prediction of cardiovascular disease and diabetes: $0 \cdot 5$ could be a suitable global boundary value". Nutrition Research Reviews 23 (2010): 247-269.

5. Munnangi S and Boktor SW. Epidemiology of study design, StatPearls Publishing, FL, USA (2017).

6. NHANES. National Health and Nutrition Examination Survey (NHANES) III Body Measurements (Anthropometry) Manual, CDC (2007).

7. Ashwell M. "The Ashwell Shape Chart-a public health approach to the metabolic risks of obesity". International Journal of Obesity 22 (1998): S213.

8. Kyle GU., et al. "Bioelectrical impedance analysis--part I: review of principles and methods". Clinical Nutrition 23.5 (2004): 1226-1243.

9. Snehalatha C., et al. "Cutoff values for normal anthropometric variables in Asian Indian adults”. Diabetes Care 26.5 (2003): 1380-1384.

10. Garaulet M., et al. "Body fat distribution in pre-and post-menopausal women: metabolic and anthropometric variables". Journal of Nutrition and Health Aging 6 (2012): 123-126. 
11. Katzmarzyk PT., et al. "Racial differences in abdominal depotspecificadiposity in white and African American adults". The American Journal of Clinical Nutrition 91 (2010): 7-155.

12. Lovejoy JC., et al. "Abdominal fat distribution and meta-bolic risk factors: effects of race". Metabolism 45 (1996): 1119-1124.

13. Lim U., et al. "Asian women have greater abdominal and visceral adiposity than Caucasian women with similar body mass index". Nutrition and Diabetes 1 (2011): e629.

14. Park YW., et al. "Larger amounts of visceral adipose tissue in Asian Americans". Obesity Research 9 (2001): 381-387.

15. Luo J., et al. "Racial and Ethnic Differences in Anthropometric Measures as Risk Factors for Diabetes". Diabetes Care 42 (2019): 126-133.

16. Jayawardena R., et al. "High dietary diversity is associated with obesity in Sri Lankan adults: an evaluation of three dietary scores". BMC Public Health 13.314 (2013).

17. Bray G A., et al. "Relation of central adiposity and body mass index to the development of diabetes in the Diabetes Prevention Program". The American Journal of Clinical Nutrition 87.5 (2008): 1212-1218.

18. Kalra S., et al. "Measures of body fat in South Asian adults". Nutrition and Diabetes 3.5 (2013): e69-e69.

\section{Assets from publication with us}

- Prompt Acknowledgement after receiving the article

- Thorough Double blinded peer review

- Rapid Publication

- Issue of Publication Certificate

- High visibility of your Published work

Website: www.actascientific.com/

Submit Article: www.actascientific.com/submission.php

Email us: editor@actascientific.com

Contact us: +919182824667 